\title{
La Somalie en mauvais État
}

\section{Alain Gascon}

\section{OpenEdition}

\section{Journals}

Édition électronique

URL : http://journals.openedition.org/echogeo/4484

DOI : 10.4000/echogeo.4484

ISSN : 1963-1197

\section{Éditeur}

Pôle de recherche pour l'organisation et la diffusion de l'information géographique (CNRS UMR 8586)

\section{Référence électronique}

Alain Gascon, "La Somalie en mauvais État », EchoGéo [En ligne], Sur le Vif, mis en ligne le 27 mai 2008, consulté le 19 avril 2019. URL : http://journals.openedition.org/echogeo/4484 ; DOI : 10.4000/ echogeo.4484

Ce document a été généré automatiquement le 19 avril 2019

\section{(c) (i) (9)}

EchoGéo est mis à disposition selon les termes de la licence Creative Commons Attribution - Pas d'Utilisation Commerciale - Pas de Modification 4.0 International 


\title{
La Somalie en mauvais État
}

\author{
Alain Gascon
}

\section{Les vingt ans d'un conflit oublié}

1 Unis pour déloger le dictateur Siyaad Barre en 1991 et repousser les casques bleus de l'Onusom (1992-1993), les chefs de guerre somaliens n'ont jamais accepté de s'effacer devant le gouvernement de transition. Ce dernier, imposé par Ismaël Omar Guelleh, président de Djibouti, à l'issue de la réunion d'Arta (2000), a siégé au Kenya avant de rentrer, après de longues tractations, à Mogadiscio. En juin 2006, les miliciens des tribunaux islamistes l'ont chassé de la capitale au grand soulagement des habitants lassés des affrontements entre seigneurs de la guerre, violents et corrompus, par ailleurs stipendiés par les États-Unis. La population a vite déchanté : les islamistes ont imposé leur ordre moral. Ils ont séparé hommes et femmes, interdit la musique, les danses, les chants et la télévision ${ }^{1}$. Le gouvernement provisoire, menacé dans son repli de Baydhowa, a fait appel à l'Éthiopie qui, en 15 jours (décembre 2006-janvier 2007), a balayé les milices islamistes à l'aide des États-Unis et du Kenya et avec l'aval de l'Union européenne (UE) et de l'Union africaine (UA). Depuis, l'armée éthiopienne peine à se maintenir à Mogadiscio dont les miliciens ne parviennent pas à l'expulser (Gascon, 2008). Périodiquement, une attaque ou un bombardement plus meurtriers, l'enlèvement de journalistes ou d'humanitaires, un acte de piraterieou le repêchage de boat people en mer Rouge font revenir la Somalie dans une actualité dont elle sort, très vite. Déconcertée par des affrontements où les camps, tour à tour, se mêlent puis se déchirent au gré d'alliances changeantes, la communauté internationale se contente d'un service humanitaire minimum. Tout autant impuissants, les Somaliens assistent au suicide de la Somalie. Renonçant à la fiction, le romancier Nuruddin Farah a publié un livre d'enquêtes, Hier, demain, où des Somaliens, réfugiés en Afrique et en Europe, expriment leur détresse.

Il témoigne: «C'était surprenant de voir un pays entier partir en fumée dans les ventes aux enchères » (p. 18). Il constate, amer $^{2}$ : « Notre peuple est à la merci de ceux qui trafiquent en abusant de la misère humaine, des politiciens qui se comportent comme des cow-boys et qui ont divisé le pays en fiefs, et d'une cabale de criminels qui dirigent ces fiefs en prétendant avoir reçu l'investiture d'un clan 
(p. 20). Un interlocuteur affirme ensuite: "Nous sommes victimes de l'effondrement de l'État, victimes d'un accident de l'histoire» (p. 96) pour ensuite asséner: "Les guerres civiles ne requièrent pas d'explications. Elles surgissent, elles surviennent, et basta!» (p. 98).

2 Nous tenterons de rappeler de l'oubli les conflits somaliens, puis de comprendre pourquoi un État, « qui avait tout pour réussir », a échoué, entraînant, à terme, la dislocation de la Somalie.

\section{Cachez cette guerre que je ne saurai voir...}

3 Le représentant de l'Onu en Somalie déplorait récemment que l'instabilité chronique de la péninsule ne soit pas ressentie comme une menace vitale pour la paix mondiale à l'instar des conflits du grand Moyen-Orient (Palestine, Irak, Afghanistan). De plus, le souvenir cuisant de l'échec de l'Onusom retarde l'arrivée de la force africaine d'interposition, dépêchée par l'UA et censée relever les Éthiopiens qui, en l'attendant, contiennent les islamistes. Les bases françaises et états-uniennes de Djibouti surveillent le détroit de Bab el-Mandeb emprunté par les pétroliers, se gardant bien, sauf l'exception du voilier Ponant ${ }^{3}$, de s'aventurer en Somalie. La flotte internationale engagée dans la guerre d'Afghanistan protège les navires qui ravitaillent en nourriture la Somalie en guerre, mais les agences de l'ONU ont dû traiter avec les chefs de guerre pour que, moyennant rétribution, ils alimentent, la population. Après le 11 septembre, les Etats-Unis ont interdit les hawilad, les sociétés qui transféraient par fax, sous contrôle des clans, les fonds de la diaspora somalienne vers la Somalie car ils les soupçonnaient de financer Al-Qaïda (Montclos, 2001). De rares témoignages dépeignent une misère et une violence extrêmes et la population ( 9,1 millions d'habitants) victime de trafics en tout genre (armes et qaat ${ }^{4}$ ) et des affrontements entre chefs de guerre, mais faisant preuve d'une capacité opiniâtre pour survivre en l'absence d'État. La production agricole végète, il n'y a plus ni écoles ni hôpitaux ni entretien des infrastructures même si des "banques", des sociétés de téléphone portable, de transports aériens et routiers et d'innombrables milices foisonnent, disparaissent... La communauté internationale tente d'enfermer dans un cordon sanitaire ce désordre endémique lointain qu'elle ne comprend pas tant le jeu des acteurs est déroutant. Elle préfère demeurer en dehors d'un conflit dont on sait, d'expérience, qui n'est pas « soluble dans l'humanitaire. »

4 Dans les États voisins de la Somalie, Djibouti, Éthiopie et Kenya, où vivent quelque 5,5 millions de Somali ${ }^{5}$, on sait que le conflit a déjà franchi leurs frontières. À ce danger, aggravé par la déliquescence des institutions étatiques somaliennes, s'ajoute l'arrivée de milliers de réfugiés dans des régions déjà sensibles : le Nord-Est du Kenya, la région-État Somali en Éthiopie et la moitié méridionale de Djibouti. L'armée boucle l'Ogadén ${ }^{6}$ éthiopien depuis l'attaque (avril 2007), par des fronts somali islamiques locaux, d'un site de forage pétrolier d'une entreprise chinoise (avril 2007). En effet, ces indices d'hydrocarbure, connus depuis les années 1930 en territoire éthiopien, n'ont jamais été explorés, faute de moyens financiers et à cause de l'insécurité chronique de cette région frontalière. En outre, l'Éthiopie où vivent 4,4 millions de Somali $(5,8 \%$ de sa population nationale) craint que l'« abcès » somalien ne la contamine et y réveille les irrédentismes. En effet, Mogadiscio a soutenu des maquis en Ogadén jusqu'à la reconnaissance mutuelle de la frontière éthio-somalienne en 1988. En 1964 puis 1977-1978, l'armée somalienne a envahi l'Ogadén soutenue par une guérilla somali ogaadeeni (Gascon \& Hirsch, 1990). Les 
Éthiopiens gardent la mémoire du jihad de l'iman Ahmed Graññ (le Gaucher) qui, au XvI siècle, faillit emporter le royaume chrétien. Au Kenya, les Somali ( $2 \%$ de la population nationale), établis dans le North-Eastern Frontier District, ont livré une longue guérilla à l'armée régulière qui craint toujours un retour à l'insécurité.

Le président de Djibouti, Ismaël Omar Guelleh, un Somali né à Dirré Dawa en Éthiopie, n'a pas épargné ses efforts pour rétablir la paix et l'unité de la Somalie. En fait, il cherche surtout l'appui des Somali de la Corne contre son opposition et surtout contre les Afar - l'autre moitié des 830000 Djiboutiens - qui ont des sympathies pour l'Éthiopie. Ses relations sont tendues avec le Somaliland ex-britannique qui a proclamé son indépendance en 1991. Cet État, ignoré par l'ONU et l'UA, s'est reconstruit avec le seul apport financier de sa diaspora (Farah \& Lewis, 1997). On y a tenu des élections législatives et présidentielles sans provoquer le moindre incident. Djibouti, par lequel transite $90 \%$ du commerce des 77,1 millions d'Éthiopiens, redoute la concurrence du port somalilandais de Berbera (Gascon 2005). Toutefois, la géométrie de ces relations de voisinage, d'opposition et d'alliance varie au gré des changements de la conjoncture géopolitique à différentes échelles. La Somalie, jusqu'à présent dans l'arrière-cour du conflit du Moyen-Orient, risque de se retrouver en première ligne si le Yémen est gagné par l'islamisme. L'intervention éthiopienne a déjà provoqué des appels au jihad contre un État « chrétien » et des rapprochements inattendus dans la Corne. L'armée éthiopienne a produit des preuves de l'implication de combattants «arabes » et du soutien érythréen aux tribunaux islamiques par ailleurs traqués en Érythrée. La coalition engagée dans la difficile guerre contre le terrorisme peut-elle admettre que ses communications maritimes soient menacées si un pouvoir islamique s'établit sur les deux rives du détroit de Bab el-Mandeb?

\section{Un État qui avait tout pour réussir... et notamment une nation}

Indépendante depuis 1960, la Somalie n'a pas échappé au sort de bien des États d'Afrique subsaharienne: putschs militaires, parti unique, socialisme étatique, dictature, guerres civiles, déplacements de population et famines... Pourtant elle aurait dû réussir, elle avait tout pour réussir. C'était le prototype de l'État-nation, tel que les Européens l'ont défini, rêvé et idéalisé : les Somaliens professent tous l'islam sunnite chaféite, parlent à $99 \%$ le somali et placent l'élevage nomade au sommet de l'activité sociale. Pareille homogénéité culturelle (linguistique et religieuse) est unique en Afrique, domaine par excellence des « conflits ethniques.» Or, ce qui semblait être l'État-nation somalien a disparu depuis 1991, englouties dans la chute de Siyaad; la Ligue arabe doit financer ses représentations à l'étranger. Par contraste, la fuite du dictateur Mängestu n'a pas entraîné l'éclatement de l'empire éthiopien, pourtant multinational; devenu république fédérale, il a mieux résisté que la Somalie, apparemment si homogène. 


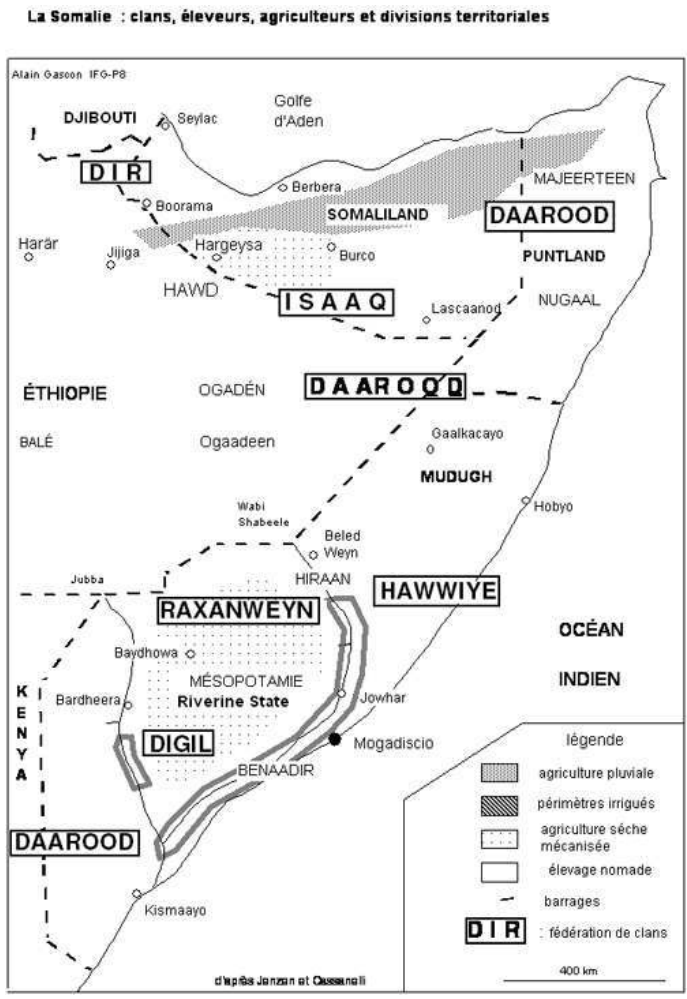

7 L'instabilité de la Somalie remonte à sa naissance même, en 1960. Les dirigeants du British Somaliland, au Nord, et la Somalia italiana, au Sud ont décidé de se fondre en un seul État. Or en 1961, lors du référendum constitutionnel, le Nord, moins peuplé, vota pour un État fédéral alors que le Sud, choisit un État unitaire. Les perdants tentèrent le premier d'une longue série de putschs, réprimés par le gouvernement de Mogadiscio. Les causes de cette divergence sont à rechercher dans les projets coloniaux et dans la période précoloniale. La Grande-Bretagne qui avait fondé le Somaliland pour éviter que la France ne s'établît en face d'Aden n'y assura qu'une présence minimale, mais s'était heurtée au Mad Mullah, Maxamed Cabdulle Xasan, défait en 1921, après vingt ans de guerre. L'Italie sut se concilier les sultans et rencontra une résistance plus faible. Dans le cadre de l'autarcie fasciste, elle lança de grands périmètres irrigués destinés à subvenir aux besoins de la métropole. Chassés de Somalie en 1941 par les Britanniques, les Italiens y revinrent en 1950 avec un mandat de dix ans l'ONU pour en préparer l'indépendance tandis que le Royaume-Uni oubliait la " boucherie d'Aden. » Les dirigeants du nouvel État ont sacrifié l'existence de la Somalie, dans configuration de 1960, au mirage de Grande Somalie. Outre qu'il portait en lui la prise du pouvoir par les militaires, ce mythe national n'évoquait rien pour les Somaliens qui avaient connu une péninsule divisée en sultanats et cités-États. Le drapeau de la république - une étoile blanche à cinq branches sur fond bleu proclamait ses revendications territoriales. La Somalie actuelle, inachevée, correspond à deux des branches, les ex-colonies italienne et britannique, auxquelles il manque trois branches: les «frères séparés" d'Éthiopie, du Kenya et de Djibouti. Sa politique extérieure belliqueuse l'isola de ses voisins où elle soutint des maquis irrédentistes. Elle constitua, à grands frais, une armée nombreuse préparant ainsi le putsch de Siyaad Barre, en 1969.

En échange de la promesse de la Grande Somalie, le général somma les Somaliens de s'en remettre à son arbitraire. À la recherche d'armement, il se tourna vers l'Union soviétique dont il devint l'allié privilégié dans la Corne. Il adopta le marxisme-léninisme, lança des 
campagnes contre l'islam et la «détribalisation » qui interdisait de mentionner toute affiliation clanique. Il nationalisa les entreprises étrangères, c'est-à-dire italiennes, et, en conséquence l'Italie ferma son marché aux produits somaliens. Le Benaadir et Mogadiscio et le bas Jubba autour de Kismaayo, qui avaient jusque-là bénéficié de l'attention du pouvoir colonial et post-colonial, périclitèrent. En 1974, dans l'espoir d'attirer les capitaux et les consommateurs d'Arabie et des États pétroliers du Golfe, le dictateur adhéra à la Ligue Arabe. En dépit du relais de l'importante diaspora somalienne, les rares investissements se résumèrent à la construction de mosquées et d'écoles coraniques dans État officiellement athée. La détribalisation désorganisa la solidarité traditionnelle alors qu'à partir de 1973 survenait la première des crises alimentaires qui frappèrent la Corne. Pendant la famine, on déplaça, de force, les éleveurs nomades du Nord pour les sédentariser, au Sud, entre les vallées du Jubba et du Wabi Shabeele dans des villages Self Help (Piguet, 1998). En 1977, sans le soutien de l'URSS, son armée enleva l'Ogadén aux Éthiopiens, alors en pleine Révolution. Mais, en 1978, l'Union soviétique et ses alliés, rangés aux côtés de l'Éthiopie, l'aidèrent battre l'armée somalienne en dépit des appels que Siyaad aux États-Unis.Cette déroute dessilla les yeux des Somaliens. Des officiers entrèrent alors en dissidence puis en rébellion au Somaliland et au Puntland (la pointe de la péninsule) et se réfugièrent en Éthiopie. Pendant la guerre d'Ogadén, le Nord, plus exposé, avait souffert des opérations militaires et accueilli un grand nombre de réfugiés. Mais c'est par Mogadiscio, qui prélevait sa dîme au passage, que parvenait l'aide internationale.

La défaite entraîna l'effondrement de l'économie étatisée et une dérive clanique et kleptocratique. Son pouvoir peu à peu réduit à la capitale, le président, «maire de Mogadiscio ", octroya à sa famille et son clan les revenus de l'État, incapable désormais de payer les salaires et laissa ses familiers dépecer le territoire national. Toutefois, l'excolonie britannique connaissait une embellie économique après de longues années de catastrophes. Les négociants exportaient en masse, vers la péninsule Arabique des carcasses bovines pour répondre aux énormes besoins au moment du hajj à La Mecque. Au milieu des années 1980, ces ventes représentaient en moyenne $90 \%$ des recettes à l'exportation de la Somalie.Les éleveurs et les commerçants, craignant la rapacité de Siyaad rejoignirent le mouvement séparatiste formé par des officiers rebelles hébergés par l'Éthiopie. En 1988, pressé par l'Italie, agissant pour l'UE, le président renonça à la Grande Somalie et fit la paix avec Mängestu. Les deux dictateurs, aux abois, reconnurent leur frontière commune et cessèrent l'aide aux fronts séparatistes. Siyaad lança alors son armée contre l'ex-Somaliland : cette agression souda les opposants, jusque-là divisés, qui, après trois ans de combats, chassèrent le général. Contrairement à ce qui est advenu en Éthiopie, la chute de la dictature a englouti l'État et n'a pas apporté la paix.

\section{Vers la disparition inéluctable de l'État en Somalie?}

L'échec de l'État-nation somalien est souvent imputé au clanisme invétéré des Somali intrinsèquement inconciliable avec le modèle de l'État importé par les colonisateurs. Or, anthropologues et historiens somalisants hésitent maintenant à généraliser à toute la péninsule, les conclusions des travaux effectués au Somaliland par I.M. Lewis et exposés dans : A Pastoral Democracy (Lewis, 1961,1982 ; Djama, 1997). À la fin du XIXe siècle, lorsque les Européens se partagèrent la péninsule somali, ce n'était pas une terra incognita des États: des sultanats, des émirats et des cités-États dont les territoires recoupaient les 
divisions claniques auxquelles échappaient les nombreux hors clans (esclaves, minorités, castes). À l'intérieur des fédérations de clans, les clans d'éleveurs ont la prééminence sur les clans d'agriculteurs, traditionnellement mis à contribution lors des disettes. L'adoption des hors clans était une forme de dépendance. Le déracinement des populations et la dissolution des structures d'un État qui avait prétendu remplacer les lignages et l'islam par le parti unique a contribué à revitaliser le pouvoir des chefs traditionnels et des cheikhs. L'appartenance clanique est devenue l'un des moyens, avec les confréries musulmanes, de se lier à ceux qui ont capté et privatisé les pouvoirs régaliens de l'État défunt. Nuruddin Farah assimile le clan business (sic.) à des associations mafieuses. L'islam somali, souvent présenté comme un facteur d'unité, notamment face aux chrétiens, est traversé de divisions profondes. La péninsule a connu de nombreux et violents jihad locaux où s'opposèrent des confréries rivales qui se déchirèrent au cours des combats de Maxamed Cabdulle Xasan.

11 En Somalie, la colonisation a supprimé, sans toutefois les abolir complètement, les configurations politiques et les identités préexistantes tout en créant d'autres découpages et appartenances. Les administrations coloniales ont tantôt combattu, rallié ou stipendié les sultans, les chefs de clans et les cheikhs tout en recrutant des troupes supplétives. La république, avant la dictature, essaya de se concilier les chefs traditionnels en les faisant entrer dans les partis politiques. Cet "entrisme», ces compromissions, qui les déconsidérèrent, fournirent un argument de choix à Siyaad quand il les supprima. Les colonisateurs ont également spécialisé chaque territoire dans une production particulière : au Nord, l'élevage d'exportation vers l'Arabie et au Sud, les grandes fermes irriguées tournées vers le marché métropolitain. Les gouvernements somaliens n'ont guère apporté de changement à ces choix. De plus, le jeune État s'est épuisé en mobilisant et en militarisant toutes ses ressources pour atteindre le mirage de la Grande Somalie. Les épreuves subies par la Somalie indépendante ont élargi la fracture identitaire et territoriale, héritée de la colonisation, et la faillite totale de la dictature a conduit au discrédit puis à l'éclatement de l'État. Une communauté de langue, d'histoire, de culture et de religion ne suffit pas à assurer la viabilité d'un État, fût-il homogène.

\section{«Somalisation » et balkanisation}

Peut-on dire que l'État a disparu en Somalie ? Il se survit dans le Somaliland qui s'est reconstruit à l'intérieur des limites coloniales, en regroupant des clans de fédérations différentes et sur la base économique de l'élevage d'exportation. Doit-on attendre que, par scissiparité, d'autres États se partagent la péninsule somali comme le Puntland, à la pointe de la Corne? D'autres régions aspirent à l'existence étatique comme le Mudug, le Riverine State ou le bas-Jubba. Plutôt qu'une base clanique, ces embryons d'États comprennent un port principal avec un hinterland de producteurs et de consommateurs. Peut-on envisager la "somalisation» de la Corne de l'Afrique comme on a parlé de la balkanisation de l'Europe centrale? C'est pour éviter le partage-somalisation de la péninsule que l'ONU refuse, sous la pression de l'UA et de la Ligue Arabe, de reconnaître le Somaliland. Djibouti, l'Érythrée et l'Égypte redoutent qu'il n'offre un nouveau débouché à l'Éthiopie enclavée et accroisse son poids dans la région. Les États arabes craignent pour l'« arabisation » des Somaliens qui, sous Siyaad, ont officiellement adopté le somali écrit en caractères latins et dont l'islam conserve des traits jugés " hérétiques " par les fondamentalistes. Ils veulent tenir fermement les deux rives du détroit de Bab el- 
Mandeb. Somalisation pourrait aussi signifier que des autorités issues de la société civile prennent en charge les pouvoirs régaliens de l'État. En effet, à la faveur d'un apaisement relatif des combats au milieu des années 2000, les hommes d'affaires somaliens, en relation avec les diasporas, ont relancé les activités du secteur tertiaire dans la capitale et le Benaadir et payé les chefs de guerre afin qu'ils les protègent. Or, leurs protecteurs étant inefficaces, ils ont appelé les milices islamistes à Mogadiscio entraînant l'intervention éthiopienne.

Quelle(s) étiquette(s) apposer sur le(s) conflit(s) en Somalie: guerre civile, conflits de clans, guerre des gangs, jihad, séparatismes, éleveurs contre agriculteurs, lutte de «la croix contre le croissant ", guerre contre le terrorisme? Toutes sans doute. En effet, au cours de l'enchaînement tragique qui a abouti à la catastrophe actuelle, les affrontements ont, tour à tour et simultanément, pris toutes ces significations.

\section{BIBLIOGRAPHIE}

Central Statistical Agency, Ethiopia. Statistical Abstract 2006, Addis Ababa, 2006.

DJAMA Marcel, « Trajectoire du pouvoir en pays somali », M. Djama et A. Gascon (dir.), La Corne dans tous ses États, Cahiers d'Études Africaines, XXXVII (2), 146, 1997, p. 403-428.

FARAH Ahmed Y. et LEWIS Ioan M., « Making Peace in Somaliland », M. Djama et A. Gascon (dir.), La Corne dans tous ses États, Cahiers d'Études Africaines, XXXVII (2), 146, 1997, p. 349-377.

GASCON Alain, «L'intervention éthiopienne en Somalie : la croix contre le croissant? », OutreTerre, 2008 (à paraître).

GASCON Alain, « Djibouti : Singapour sur mer Rouge. Un confetti d'Empire futur dragon africain », Outre-Terre, Revue française de géopolitique, De l'Afrique au Gondwana?, nº 11, juin, 2005,

p. 451-466.

GASCON Alain, «Les Somali. Nomadisme, migrations et déplacements forcés, Populations réfugiées. De l'exil au retour, L. Cambrézy et V. Lassailly-Jacob (dir.), Paris, Éditions de l'IRD, 2001, p. 77-94.

GASCON Alain et HIRSCH Bertrand, « Naissance d'une frontière. Un siècle de conflit somaloéthiopien ", Colloque : La géopolitique des diplomates, des militaires et des professeurs (Paris 26-27-28 mai 1990), Paul Claval (dir.), 1990, 12 p. et cartes (communication non publiée)

LAITIN D. et SAMATAR S., Somalia, Nation in Search of a State, Boulder-Londres, Westview PressGower, 1987.

LEWIS IOan M.,A Modern History of Somalia, Nation and State in the Horn of Africa. Harlow, Longman, 1982.

LEWIS IOan M.,A Pastoral Democracy, Londres, International African Institute, Oxford University Press, 1961.

NURUDDINH FARAH, Hier, demain. Voix et témoignages de la diaspora somalienne, Paris, Le Serpent à plumes, 2001. 
PÉROUSE DE MONTCLOS Marc-Antoine, « Réseaux financiers et hawilad. Le rôle de la diaspora somalienne dans la reconstruction du pays ", Populations réfugiées. De l'exil au retour, L. Cambrézy et V. Lassailly-Jacob (dir.), Paris, Éditions de l'IRD, 2001, p. 95-118.

PIGUET François, Des nomades entre la ville et les sables. La sédentarisation dans la Corne de l'Afrique, Paris-Genève, Karthala-IUED, 1998.

Population et Sociétés, « Tous les pays du monde (2007) », n 436, juillet-août 2007.

Communication non-publiée

\section{NOTES}

1. Au moment de la retransmission de la Coupe du monde de football !

2. Invité à l'EHESS en mai 2006, il a encore radicalisé sa position : il déclara, en substance, que « ses dirigeants avaient déclaré la guerre au peuple somalien » (témoignage personnel).

3. . Article écrit après la libération de l'équipage et des passagers du voilier français.

4. . Stupéfiant « léger»: Catha edulis Forsk. Tchat en amharique (éthiopien); khât en arabe.

5. . Somalien désigne les citoyens de la République somalienne et Somali les citoyens, de langue somali, de Djibouti, du Kenya et de la région-État Somali d'Éthiopie.

6. . Ogadén désigne une région éthiopienne et Ogaadeen un clan somali centré sur cette même région.

7. Je dois cette information à Marcel Djama (CIRAD) que je remercie ici.

\section{RÉSUMÉS}

La Somalie a sombré depuis 1988 dans une guerre civile que rien ne semble pouvoir arrêter. Les chefs de guerre se disputent des portions du territoire en autant de repaires pour y organiser leurs trafics criminels. Expulsés de Mogadiscio par les milices des tribunaux islamiques, ils y sont revenus avec le gouvernement provisoire, réinstallé par l'Éthiopie. Toutefois, au Somaliland, au Nord, les autorités séparatistes ont rétabli la paix sur tout leur territoire, reconstruit les infrastructures et organisé des élections que personne n'a contestées, mais l'ONU, sous la pression conjuguée de la Ligue Arabe et de l'Union africaine, refuse de reconnaitre l'indépendance de l'ex-colonie britannique. Après vingt ans d'indifférence à ce lointain conflit, l'État somalien est menacé de disparition.

Since 1988 Somalia has sunk into an endless civil war. Warlords have been fighting over parts of the national territory in order to expand their criminal traffics. They were expelled from the capital by the Islamic court militias but they have returned to it when the Provisionnal Government of Somalia was restored by Ethiopia in Mogadisico. Under the pressures of the Arab League and of the African Union the United Nations have constantly refused to recognize the independence of Somaliland, a former British colony, despite its separatist government has brought peace on all their territory, rebuilt equipments and held fair elections. Twenty years of indifference to this remote conflict have threatened the Somalian state to death. 
INDEX

Mots-clés : Somalie, Corne de l'Afrique, guerre civile, organisations internationales

Keywords : Somalia, Horn of Africa, civil war, international institutions

\section{AUTEUR}

\section{ALAIN GASCON}

Alain Gascon (며gascon@noos.fr) est professeur à l'Institut français de géopolitique de l'Université Paris 8, chargé de cours de civilisation au Département Afrique de l'INALCO et associé au Centre d'études africaines (EHESS/IRD). Il travaille sur les recompositions territoriales et identitaires parmi les populations d'agriculteurs des hautes terres et d'éleveurs des basses terres en Éthiopie et dans la Corne de l'Afrique (Érythrée, Djibouti, Somalie) en proie aux crises politiques, sociales et environnementales. 